\title{
On simultaneous diophantine equations
}

by

Shin-IChi Katayama (Tokushima) and Claude Levesque (Québec)

1. Introduction. Let $a$ and $b$ be distinct positive integers which are not perfect squares. In [Be1], M. A. Bennett investigated the number of integer solutions of the following simultaneous diophantine equations:

$$
\left\{\begin{array}{l}
x^{2}-a z^{2}=u, \\
y^{2}-b z^{2}=v,
\end{array}\right.
$$

where $u$ and $v$ are nonzero integers with $a v \neq b u$. He gave more precise results for the following specific equations:

$$
\left\{\begin{array}{c}
x^{2}-a z^{2}=1, \\
y^{2}-b z^{2}=1,
\end{array}\right.
$$

which are often called simultaneous Fermat-Pell equations. Without loss of generality, we may consider the solvability of (1.1) and (1.2) only in positive integers. We will denote the number of positive integer solutions of (1.1) by $N_{+}(a, b, u, v)$.

M. A. Bennett $[\mathrm{Be} 1]$ proved that

$$
N_{+}(a, b, 1,1) \leq 3,
$$

and that in general

$$
N_{+}(a, b, u, v) \ll 2^{\min \{\omega(u), \omega(v)\}}(\log |u|+\log |v|),
$$

where the implied constant is absolute, $\omega(m)$ being the number of distinct prime factors of $m$. In $[\mathrm{M}-\mathrm{R}], \mathrm{D}$. W. Masser and J. H. Rickert gave a specific infinite parametrized family of $(a, b)$ with $N_{+}(a, b, 1,1) \geq 2$. M. A. Bennett (see [Be1] and $[\mathrm{Be} 2])$ suggested that $N_{+}(a, b, 1,1) \leq 1$ except for those couples $(a, b)$ (for which he assumed $a, b$ squarefree and $b>a \geq 2$ ).

In this paper, we shall investigate the number of solutions of the following simultaneous diophantine equations:

2000 Mathematics Subject Classification: 11D25, 11D45. 


$$
\left\{\begin{array} { r l } 
{ x ^ { 2 } - ( M ^ { 2 } + 4 ) d z ^ { 2 } } & { = M ^ { 2 } , } \\
{ y ^ { 2 } - d z ^ { 2 } } & { = 1 , }
\end{array} \quad \text { i.e., } \quad \left\{\begin{array}{rl}
x^{2}-\left(M^{2}+4\right) y^{2} & =-4, \\
y^{2}-d z^{2} & =1,
\end{array}\right.\right.
$$

where $M$ is assumed to be an odd positive integer and where, without loss of generality, we may assume $d$ to be a squarefree integer.

Any natural number $n$ can be written in the form $n_{1} n_{2}^{2}$, where $n_{1}$ is squarefree. We write

$$
s(n)=n_{1} \quad \text { and } \quad q(n)=n_{2},
$$

and following [W1] we call $s(n)$ the square class of $n$.

For any positive integer $M$ and $e \in\{1,-1\}$, we define two binary recurrence sequences $g_{n}(M, e)$ and $h_{n}(M, e)$ by

$$
\begin{aligned}
& g_{n+2}(M, e)=M g_{n+1}(M, e)-e g_{n}(M, e), \\
& h_{n+2}(M, e)=M h_{n+1}(M, e)-e h_{n}(M, e),
\end{aligned}
$$

with initial terms $g_{0}(M, e)=2, g_{1}(M, e)=M$ and $h_{0}(M, e)=0, h_{1}(M, e)=1$. In the following, we will consider only the case $e=-1$ unless it is clearly stated. If there is no risk of confusion, we will simply write $g_{n}$ and $h_{n}$ for $g_{n}(M, e)$ and $h_{n}(M, e)$. When $M=1$ and $e=-1,\left\{h_{n}\right\}_{n \in \mathbb{N}}$ is the classical Fibonacci sequence and $\left\{g_{n}\right\}_{n \in \mathbb{N}}$ is the usual Lucas sequence.

The purpose of this paper is to show that (1.3) has at most one positive integer solution when $\omega(d) \leq 4$. More precisely, we will prove the following theorem, in which by definition,

$$
d_{k}=s\left(h_{2 k+1}^{2}-1\right) .
$$

In particular, the first values of $d_{k}$ are the following ones:

$$
\begin{aligned}
d_{1}= & s\left(M^{2}+2\right) \\
d_{2}= & s\left(\left(M^{2}+1\right)\left(M^{2}+2\right)\left(M^{2}+3\right)\right) \\
d_{3}= & s\left(\left(M^{2}+1\right)\left(M^{2}+2\right)\left(M^{2}+3\right)\left(M^{4}+4 M^{2}+2\right)\right), \\
d_{4}= & s\left(\left(M^{2}+2\right)\left(M^{4}+4 M^{2}+2\right)\left(M^{4}+3 M^{2}+1\right)\left(M^{4}+5 M^{2}+5\right)\right), \\
d_{5}= & s\left(\left(M^{2}+1\right)\left(M^{2}+2\right)\left(M^{2}+3\right)\left(M^{4}+3 M^{2}+1\right)\right. \\
& \left.\times\left(M^{4}+4 M^{2}+1\right)\left(M^{4}+5 M^{2}+5\right)\right) \\
d_{6}= & s\left(\left(M^{2}+1\right)\left(M^{2}+2\right)\left(M^{2}+3\right)\left(M^{4}+4 M^{2}+1\right)\right. \\
& \left.\times\left(M^{6}+5 M^{4}+6 M^{2}+1\right)\left(M^{6}+7 M^{4}+14 M^{2}+7\right)\right) .
\end{aligned}
$$

THEOREM 1.1. Let $d$ be a positive squarefree integer with $\omega(d) \leq 4$ and suppose $M$ to be an odd positive integer.

(i) For $M \in\{1,3,5\}$, the simultaneous diophantine equations in (1.3) have no integer solution except for 


$$
d= \begin{cases}3,6,42,55,377,1155 & \text { when } M=1, \\ 11,330 & \text { when } M=3, \\ 3,546 & \text { when } M=5\end{cases}
$$

where in each case there is exactly one positive integer solution.

(ii) Suppose $M \neq 1,3,5$ and let $\mathcal{T}=\left\{d_{1}, d_{2}, d_{3}, d_{4}\right\}$. If $d \notin \mathcal{T}$, then the simultaneous diophantine equations in (1.3) have no positive integer solution. If (1.3) has a positive solution, then there exists $k \in\{1,2,3,4\}$ such that $d=d_{k}$ and the solution is uniquely given by

$$
x=g_{2 k+1}, \quad y=h_{2 k+1}, \quad z=q\left(h_{2 k+1}^{2}-1\right) .
$$

Our results generalize those of P. G. Walsh in the following sense: P. G. Walsh [W1] considered the case where $M=2$ in (1.3), namely,

$$
\left\{\begin{array} { r l } 
{ x ^ { 2 } - 8 d z ^ { 2 } } & { = 4 , } \\
{ y ^ { 2 } - d z ^ { 2 } } & { = 1 , }
\end{array} \quad \text { i.e., } \quad \left\{\begin{array}{c}
w^{2}-2 d z^{2}=1, \\
y^{2}-d z^{2}=1 .
\end{array}\right.\right.
$$

As far as we are concerned, we deal with the case where $M$ is any odd integer. Note that in the special case $M=1$, Theorem 1.1 takes care of the simultaneous Fermat-Pell equations

$$
\left\{\begin{array}{c}
x^{2}-5 d z^{2}=1 \\
y^{2}-d z^{2}=1
\end{array}\right.
$$

Let us mention in passing that P. G. Walsh obtained in [W2] a family of couples $(a, b)$ for which (1.2) has only the trivial solution $(x, y, z)=(1,1,0)$.

Under a very strong hypothesis, we can prove the following.

TheOREM 1.2. Assume the ABC conjecture. For any positive integer $M$ (odd or even), there exists $d(M)$, such that for any squarefree $d>d(M)$, the simultaneous diophantine equations in (1.3) have at most one positive integer solution. Moreover, if $(x, y, z)$ is a positive integer solution of the above simultaneous diophantine equations with $d>d(M)$, then on the one hand, $x=g_{2 k+1}, y=h_{2 k+1}, z=q\left(h_{2 k+1}^{2}-1\right)$ for some $k$, and on the other hand, the algebraic integer $y+z \sqrt{d}$, of norm +1 , is the fundamental unit of the real quadratic field $\mathbb{Q}(\sqrt{d})$.

Corollary 1.3. Assume the $A B C$ conjecture. If the fundamental unit $\varepsilon_{d}$ of the real quadratic field $\mathbb{Q}(\sqrt{d})$ has norm -1 , then the simultaneous diophantine equations in (1.3) have no positive integer solution except for finitely many $d$.

2. Preliminary results. For any natural numbers $a$ and $b$, we will use the notation $a \sim b$ to mean $s(a)=s(b)$, i.e., $a b$ is a square.

Let us recall the following fundamental properties of $g_{n}$ and $h_{n}$ (see Propositions $2.1,2.2,4.5,4.6,4.7$ and 4.8 of $[\mathrm{K}-\mathrm{L}-\mathrm{N}])$. 
Lemma 2.1. Let $M$ be odd. For $n \geq 1$, we have:

(i) $\left(g_{n}, g_{n+1}\right)=1$,

(ii) $\left(h_{n}, h_{n+1}\right)=1$,

(iii) $\left(g_{n}, h_{n+1}\right)=\left(g_{n}, h_{n-1}\right)= \begin{cases}1 & \text { if } n \text { is even, } \\ M & \text { if } n \text { is odd, }\end{cases}$

(iv) $\left(g_{n}, h_{n}\right)=\left(h_{n}, 2\right)= \begin{cases}1 & \text { if } 3 \nmid n, \\ 2 & \text { if } 3 \mid n,\end{cases}$

(v) $h_{2 n+1}^{2}-1=h_{2 n} h_{2 n+2}$,

(vi) $h_{2 n}=g_{n} h_{n}$.

LemMA 2.2. Let $M$ be odd and let $r \geq 1$.

(i) If $M=1$, then

$$
g_{r} \sim 1(=M) \Leftrightarrow r=1 \text { or } 3, \quad g_{r} \sim 2(=2 M) \Leftrightarrow r=6 .
$$

(ii) If $M=3$, then $g_{r} \nsim 2, g_{r} \not 2 M$, and

$$
g_{r} \sim 1 \Leftrightarrow r=3, \quad g_{r} \sim M \Leftrightarrow r=1 .
$$

(iii) If $M=5$, then $g_{r} \not 1, g_{r} \not 2 M$, and

$$
g_{r} \sim 2 \Leftrightarrow r=6, \quad g_{r} \sim M \Leftrightarrow r=1 .
$$

(iv) Elsewhere, $g_{r} \not 2, g_{r} \nsim 2 M$, and

$$
\left.g_{r} \sim 1 \Leftrightarrow r=1 \text { (with } M \sim 1\right), \quad g_{r} \sim M \Leftrightarrow r=1 .
$$

LEMmA 2.3. Let $M$ be odd and let $r \geq 1$.

(i) If $M=1$, then

$h_{r} \sim 1(=M) \Leftrightarrow r=1,2$ or $12, \quad h_{r} \sim 2(=2 M) \Leftrightarrow r=3$ or 6.

(ii) If $M=M_{0}^{2}$ with $M_{0}>1$, then $h_{r} \not 2, h_{r} \not 2 M$, and

$$
h_{r} \sim 1(\sim M) \Leftrightarrow r=1 \text { or } 2 .
$$

(iii) If $M=\sqrt{2 M_{0}^{2}-1}>1$, then $h_{r} \not 2 M$, and

$$
h_{r} \sim 1 \Leftrightarrow r=1, \quad h_{r} \sim 2 \Leftrightarrow r=3, \quad h_{r} \sim M \Leftrightarrow r=2 .
$$

(iv) Elsewhere, $h_{r} \nsim 2, h_{r} \nsim 2 M$, and

$$
h_{r} \sim 1 \Leftrightarrow r=1, \quad h_{r} \sim M \Leftrightarrow r=2 .
$$

We want to secure the fact that for $k \geq 5$ and for $M \neq 1,3,5, d_{k}$ has at least 5 distinct prime factors. For this, we will need the following result.

Proposition 2.4. Let $M=\sqrt{2 M_{0}^{2}-1}>1$. Then

$$
s\left(g_{6}\right)=s\left(\left(M^{2}+2\right)\left(M^{4}+4 M^{2}+1\right)\right)
$$

contains at least two odd primes. 
Proof. Since $M$ is odd, and since $M^{4}+4 M^{2}+1 \equiv 6(\bmod 16)$, we respectively have

$$
M^{2}+2 \not 1 \text { nor } 2, \quad M^{4}+4 M^{2}+1 \not 1 \text { nor } 2 .
$$

Moreover, since $3 \nmid M_{0}$, we have $M^{2}+2=2 M_{0}^{2}+1 \equiv 0(\bmod 3)$, so

$$
\left(M^{2}+2, M^{4}+4 M^{2}+1\right)=\left(M^{2}+2,\left(M^{2}+2\right)^{2}-3\right)=3 .
$$

Now it is obvious that $s\left(g_{6}\right)$ contains at least two odd primes when $s\left(M^{2}+2\right)$ contains at least two odd primes $p, q$ with $p \neq 3$ and $q \neq 3$. We can then suppose that $s\left(M^{2}+2\right)$ does not contain two odd primes $p, q$ both strictly greater than 3 . Then one can easily see that the odd integer $M^{2}+2$ must be of one of the following three forms:

$$
M^{2}+2=\left\{\begin{array}{l}
9 p y^{2} \quad(\text { with } p \neq 3), \\
3 y^{2}, \\
3 p y^{2} \quad(\text { with } p \neq 3)
\end{array}\right.
$$

for some integer $y$.

Assume first $M^{2}+2=9 p y^{2}$ with $p \neq 3$. We can conclude from (2.1) and (2.2) that $s\left(g_{6}\right)$ contains 3 and $p \neq 3$.

Suppose next that $M^{2}+2=3 y^{2}$, where by hypothesis $M^{2}-2 M_{0}^{2}=-1$. Since $M>1$, we know that $M=g_{2 k+1}(2,-1) / 2$ for some $k \geq 1$, where $g_{0}=2, g_{1}=2, g_{2}=6, g_{3}=14, \ldots, g_{s+1}=2 g_{s}+g_{s-1}$, and one easily proves the following two formulas:

$$
g_{s}-8(-1)^{s}=g_{s-1} g_{s+1}, \quad\left(g_{s}, g_{s+2}\right)=2 \quad \text { for } s \geq 1 .
$$

We deduce that $M^{2}+2$ is the product of two coprime integers:

$$
M^{2}+2=\frac{g_{2 k+1}^{2}+8}{4}=\left(\frac{g_{2 k}}{2}\right)\left(\frac{g_{2 k+2}}{2}\right) .
$$

Since $M^{2}+2=3 y^{2}$, either $g_{2 k} / 2 \sim 1$ or $g_{2 k+2} / 2 \sim 1$. Writing $w^{2}=g_{2 k} / 2$ or $g_{2 k+2} / 2$, we conclude that $w^{4}-2 z^{2}=1$ for some integer $z$; this forces $w^{2}+1$ or $w^{2}-1$ to be a square, whereupon $w=1$ and $z=0$, which contradicts $M>1$.

Finally, suppose $M^{2}+2=3 p y^{2}$ with $p \neq 3$. From (2.3), we deduce that at least one of $g_{2 k}$ and $g_{2 k+2}$ must be $\sim 2$ or 6 . We saw above that $g_{2 k} \nsim 2$ and $g_{2 k+2} \nsim 2$, whereupon

$$
\text { either } \frac{g_{2 k}}{2} \sim 3 \quad \text { or } \quad \frac{g_{2 k+2}}{2} \sim 3 .
$$

So there exist positive integers $A$ and $B$ such that $9 A^{4}-2 B^{2}=1$. Using Corollary 1.3 of [B-W], we obtain $A=1, Y=2$, i.e., $M=7$, whereupon $s\left(g_{6}\right)=2 \cdot 17 \cdot 433$ contains at least two odd primes. 
Proposition 2.5. Let $M$ be an odd integer different from 1,3, 5 and let $k \geq 5$. Then $d_{k}=s\left(h_{2 k+1}^{2}-1\right)$ has at least 5 distinct prime factors.

Proof. Suppose $k=4 m+r$ for $0 \leq r \leq 3$. Then for $m \geq 1$, we have

$$
h_{2 k+1}^{2}-1 \sim \begin{cases}g_{4 m}\left(g_{2 m} \frac{h_{2 m}}{M}\right) \frac{g_{4 m+1}}{M} h_{4 m+1} & \text { if } r=0, \\ \frac{g_{4 m+1}}{M} h_{4 m+1} g_{4 m+2}\left(\frac{g_{2 m+1}}{M} h_{2 m+1}\right) & \text { if } r=1, \\ g_{4 m+2}\left(\frac{g_{2 m+1}}{M} h_{2 m+1}\right) \frac{g_{4 m+3}}{M} h_{4 m+3} & \text { if } r=2, \\ \frac{g_{4 m+3}}{M} h_{4 m+3} g_{4 m+4}\left(g_{2 m+2} \frac{h_{2 m+2}}{M}\right) & \text { if } r=3,\end{cases}
$$

i.e., $h_{2 k+1}^{2}-1 \sim A_{1} A_{2} A_{3} A_{4} A_{5}$, a product of five factors in the same order as in (2.4) with $\left(A_{i}, A_{j}\right)=1$ or 2 for $1 \leq i<j \leq 5$.

Let $M \neq \sqrt{2 M_{0}^{2}-1}$ for any $M_{0}$ and $M \neq 1,3,5$. Then from Lemmas 2.2 and 2.3 , one can verify $A_{i} \nsim 1$ nor 2 for any $1 \leq i \leq 5$ and for any $k \geq 5$. Thus we see that in this case $\omega\left(d_{k}\right) \geq 5$ for $k \geq 5$.

Let $M=\sqrt{2 M_{0}^{2}-1}>1$. Then from Lemmas 2.2 and 2.3 , one can also verify $A_{i} \not 1$ nor 2 for any $1 \leq i \leq 5$ and for any $k \geq 7$. In the cases $k=5$ and 6 , one sees

$$
\begin{aligned}
& d_{5}=s\left(h_{11}^{2}-1\right) \sim\left(g_{5} / M\right) h_{5} g_{6}\left(g_{3} / M\right) h_{3}, \\
& d_{6}=s\left(h_{13}^{2}-1\right) \sim\left(g_{7} / M\right) h_{7} g_{6}\left(g_{3} / M\right) h_{3},
\end{aligned}
$$

where $h_{3} \sim 2$ and otherwise $A_{i} \not 1$ nor 2. Moreover, from Proposition 2.4, we know that $s\left(g_{6}\right)$ contains at least two odd primes, which completes the proof.

3. Proof of the main result. Suppose now that $(x, y, z)$ is a positive integer solution of (1.3). One sees that $x=g_{2 k+1}$ and $y=h_{2 k+1}$ for some $k>0$ and $d z^{2}=h_{2 k+1}^{2}-1=h_{2 k} h_{2 k+2}=g_{k} h_{k} g_{k+1} h_{k+1}$ by Lemma 2.1. In order to make the proof easier to read, we will proceed in steps.

CAsE A. Firstly, we consider the case $M=1$. Then the assumption $\omega(d) \leq 4$ implies that at least one of $h_{s}$ or $g_{s}$ which appears in the decomposition (2.4) must be $\sim 1$ or 2 , which forces $s \leq 12$, and $2 k+1 \leq 49$. Since

$$
\begin{gathered}
h_{3}^{2}-1=3, \quad h_{5}^{2}-1=6 \cdot 2^{2}, \quad h_{7}^{2}-1=42 \cdot 2^{2}, \\
h_{9}^{2}-1=1155, \quad h_{11}^{2}-1=55 \cdot 12^{2}, \quad h_{13}^{2}-1=377 \cdot 12^{2},
\end{gathered}
$$

and since $\omega\left(s\left(h_{2 k+1}^{2}-1\right)\right) \geq 5$ for all $k$ with $7 \leq k \leq 24$, we have proved Theorem 1.1 in this case. 
Case B. Next, we consider the case $M=3$. Then from Lemmas 2.2 and 2.3 , we see that one of $g_{s}$ and $h_{s}$, which appear in the decomposition (2.4), must satisfy $s \leq 3$ and so $2 k+1 \leq 13$. Since $h_{3}^{2}-1=11 \cdot 3^{2}, h_{5}^{2}-1=330 \cdot 6^{2}$ involve the only indices $k$ with $\omega\left(s\left(h_{2 k+1}^{2}-1\right)\right) \leq 4$, we can conclude.

Case C. Let us treat the case $M=5$. From Lemmas 2.2 and 2.3, one of $g_{s}$ and $h_{s}$, which appear in the decomposition (2.4), must satisfy $s \leq 6$ and hence $2 k+1 \leq 25$. Since $h_{3}^{2}-1=3 \cdot 13^{2}, h_{5}^{2}-1=546 \cdot 30^{2}$ involve the only indices $k$ with $\omega\left(s\left(h_{2 k+1}^{2}-1\right)\right) \leq 4$, we have the required result.

Case D. Next we shall treat the case $M \neq 1,3,5$. From Proposition 2.5, we see that $\omega\left(s\left(d_{k}\right)\right) \geq 5$ for any $k \geq 5$. One can also easily verify that $d_{i} \neq d_{j}$ for any $1 \leq i<j \leq 4$. Hence the conclusion.

REMARK 3.1. In Theorem 1.1, one can determine the exceptional $d$ satisfying $\omega(d) \leq 4$ and such that $N_{+}\left(\left(M^{2}+4\right) d, d, M^{2}, 1\right)=1$ for any odd integer $M$. For example, when $M=7$, then the exceptional $d$ 's are $d=51=3 \cdot 17$ and $d=1326=2 \cdot 3 \cdot 13 \cdot 17$. One can also verify that for any odd integer $1 \leq M \leq 1827$, the simultaneous diophantine equations of (1.3) have at least one exceptional $d$ such that $\omega(d) \leq 4$ and $N_{+}\left(\left(M^{2}+4\right) d, d, M^{2}, 1\right)=1$ and there is no exceptional case for $M=1829$.

REMARK 3.2. It looks like there are not so many small odd integers $M$ for which $d \in \mathcal{T}=\left\{d_{1}, d_{2}, d_{3}, d_{4}\right\}$ with $\omega(d) \geq 5$ : we verified with a computer that the only odd integers $M, 1 \leq M \leq 5369$, such that $\omega\left(s\left(d_{1}\right)\right)=$ $\omega\left(s\left(M^{2}+2\right)\right) \geq 5$ are the following twelve:

$1829,2203,2285,2863,3121,3509,3787,4155,4733,4903,5155,5195$.

Moreover, we can show that there exist infinitely many $M$ such that under the condition $\omega(d) \leq 4$, the simultaneous diophantine equations in (1.3) have no integer solutions. Let $p_{i}(1 \leq i \leq 5)$ be distinct odd primes such that $\left(\frac{-2}{p_{i}}\right)=1$, i.e., $p_{i} \equiv 1$ or $3(\bmod 8)$. Choose $a_{i}$ such that $a_{i}^{2} \equiv-2+p_{i}$ $\left(\bmod p_{i}^{2}\right)$ for $1 \leq i \leq 5$. By the chinese remainder theorem, there exist infinitely many odd $M$ which satisfy $M \equiv a_{i}\left(\bmod p_{i}^{2}\right)$ for $1 \leq i \leq 5$. Since $M^{2}+2$ appears in $d_{1}, d_{2}, d_{3}, d_{4}$ and since $s\left(M^{2}+2\right)$ contains at least the five primes $p_{1}, \ldots, p_{5}$, we have $N_{+}\left(\left(M^{2}+4\right) d, d, M^{2}, 1\right)=0$ for these $M$. If we choose, for example, $p_{1}=3, p_{2}=11, p_{3}=17, p_{4}=19, p_{5}=41$, then under the condition $\omega(d) \leq 4, N_{+}\left(\left(M^{2}+4\right) d, d, M^{2}, 1\right)=0$ for any positive integer $M \equiv 139106648843\left(\bmod 2 \cdot 3^{2} \cdot 11^{2} \cdot 17^{2} \cdot 19^{2} \cdot 41^{2}\right)$.

4. Using the $A B C$ conjecture. In this section, we shall estimate the number of integer solutions of simultaneous diophantine equations under the $A B C$ conjecture as stated in [La]. 
$A B C$ Conjecture. For any $\varepsilon>0$, there exists a constant $K(\varepsilon)($ depending only on $\varepsilon$ ) such that if $a, b, c$ are nonzero relatively prime integers with $a+b+c=0$, then

$$
\max \{|a|,|b|,|c|\} \leq K(\varepsilon) R^{1+\varepsilon},
$$

where $R=\operatorname{rad}(a b c)=\prod_{p \mid a b c} p$ ( $p$ denoting a prime $)$.

Using the results of $[\mathrm{R}-\mathrm{W}]$ and assuming the $A B C$ conjecture, the first author proved in [Ka] that for any fixed integer $M$ (odd or even), $s\left(h_{2 k+1}^{2}-1\right)$ $>\sqrt{h_{2 k+1}^{2}-1}$ except for finitely many indices $k$. Write $\varepsilon_{2 k+1}=h_{2 k+1}+$ $\sqrt{h_{2 k+1}^{2}-1}, \mathbb{Q}\left(\sqrt{d_{k}}\right)=\mathbb{Q}\left(\sqrt{h_{2 k+1}^{2}-1}\right)$, where $d_{k}=s\left(h_{2 k+1}^{2}-1\right)$, and put $d(M)=\max \left\{d_{k} \mid k \in \mathbb{N}\right.$ and $\varepsilon_{2 k+1}$ is not the fundamental unit of $\left.\mathbb{Q}\left(\sqrt{d_{k}}\right)\right\}$. Then with the same argument as in $[\mathrm{Ka}]$, we have the following proposition.

Proposition 4.1. Assume the ABC conjecture. For any positive integer $M$ (odd or even), there exists $d(M)$ such that for any $k$ with $d_{k}=$ $s\left(h_{2 k+1}^{2}-1\right)>d(M), h_{2 k+1}+\sqrt{h_{2 k+1}^{2}-1}$ is the fundamental unit of the real quadratic field $\mathbb{Q}\left(\sqrt{h_{2 k+1}^{2}-1}\right)$.

We note that the number of positive integer solutions of (1.3) is the number of indices $k$ such that $\mathbb{Q}(\sqrt{d})=\mathbb{Q}\left(\sqrt{h_{2 k+1}^{2}-1}\right)$ for a fixed $d$. From this proposition, we see that the real quadratic fields $\mathbb{Q}\left(\sqrt{h_{2 k+1}^{2}-1}\right)$ are all different except for finitely many indices $k$. Thus we have proved Theorem 1.2 .

Acknowledgements. The second author was supported by NSERC and FCAR grants.

\section{References}

[Be1] M. A. Bennett, On the number of solutions of simultaneous Pell equations, J. Reine Angew. Math. 498 (1998), 173-199.

[Be2] -, Solving families of simultaneous Pell equations, J. Number Theory 67 (1997), 241-251.

[B-W] M. A. Bennett and P. G. Walsh, The diophantine equation $b^{2} X^{4}-d Y^{2}=1$, Proc. Amer. Math. Soc. 127 (1999), 3481-3491.

[Ka] S.-I. Katayama, The abc conjecture and the fundamental system of units of certain real bicyclic biquadratic fields, Proc. Japan Acad. Ser. A Math. Sci. 75 (1999), 198-199.

[K-L-N] S.-I. Katayama, C. Levesque and T. Nakahara, On the unit group and the class number of certain composita of two real quadratic fields, Manuscripta Math. 105 (2001), 85-101. 
[La] S. Lang, Old and new conjectured diophantine inequalities, Bull. Amer. Math. Soc. 23 (1990), 37-75.

[M-R] D. W. Masser and J. H. Rickert, Simultaneous Pell equations, J. Number Theory 61 (1996), 52-66.

[R-W] P. Ribenboim and P. G. Walsh, The ABC conjecture and the powerful part of terms in binary recurring sequences, ibid. 74 (1999), 134-147.

[W1] P. G. Walsh, On integer solutions to $x^{2}-d y^{2}=1, z^{2}-2 d y^{2}=1$, Acta Arith. 82 (1997), 69-76.

[W2] - On two classes of simultaneous Pell equations with no solutions, Math. Comp. 68 (1999), 385-388.

Department of Mathematical Sciences

Tokushima University

Tokushima 770-8502, Japan

E-mail: katayama@ias.tokushima-u.ac.jp
CICMA

Département de Mathématiques et Statistique

Université Laval

Québec, Canada G1K 7P4

E-mail: cl@mat.ulaval.ca

Received on 28.8.2001

and in revised form on 27.11.2002 